\title{
ORIGINAL RESEARCH-ANATOMY/PHYSIOLOGY
}

\section{Associations Among Physiological and Subjective Sexual Response, Sexual Desire, and Salivary Steroid Hormones in Healthy Premenopausal Women}

\author{
Sari M. van Anders, $\mathrm{PhD},{ }^{*}$ Lori Brotto, $\mathrm{PhD},{ }^{\dagger}$ Janine Farrell, $\mathrm{BA},{ }^{\ddagger}$ and Morag Yule, $\mathrm{BA}^{\dagger}$ \\ *Departments of Psychology and Women's Studies, Neuroscience Program, University of Michigan, Ann Arbor, MI, USA;

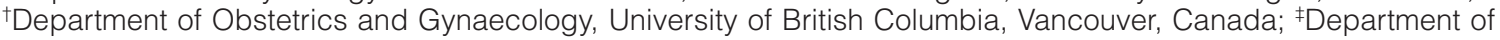 \\ Psychology, Simon Fraser University, Burnaby, Canada \\ DOI: $10.1111 / j .1743-6109.2008 .01123 \cdot x$
}

\begin{abstract}
A B S T R A C T
Introduction. Few studies have examined how sexual arousal influences healthy premenopausal women's hormones, limiting our understanding of basic physiology and our ability to transfer knowledge from clinical and nonhuman populations.

Aim. To examine how sexual arousal and steroid hormones (testosterone [T], cortisol [C], estradiol [E]) were linked, to see whether hormone levels influenced and/or changed in response to sexual arousal elicited via visual erotic stimuli in healthy women.

Methods. Participants included 40 healthy premenopausal women not using exogenous hormones.

Main Outcome Measures. Change in genital sexual arousal (vaginal pulse amplitude), change in subjective sexual arousal, sexual desire (via the Sexual Desire Inventory and Female Sexual Function Index scales), as well as T, C, and $\mathrm{E}$ via saliva samples taken before and following viewing of erotic stimuli as genital arousal was recorded via a vaginal photoplethysmograph.

Results. E increased in response to sexual stimuli but this was not statistically associated with genital sexual arousal, whereas $\mathrm{C}$ decreased in association with genital sexual arousal, and $\mathrm{T}$ showed no statistically significant change. Relationship status was linked to genital but not subjective sexual arousal such that dating women exhibited higher genital sexual arousal than single or partnered women. Results indicated that all three hormones were associated with self-reported genital arousal (via the Detailed Assessment of Sexual Arousal scales) and sexual desire in different domains, and both $\mathrm{T}$ and $\mathrm{E}$ were associated with self-reported orgasms.

Conclusion. Findings point to the need to examine multiple hormones in multiple ways (e.g., baseline, changes, stimulated) and question using erotic stimuli-induced arousal as a model for women's endocrine responses to sexuality. van Anders SM, Brotto L, Farrell J, and Yule M. Associations among physiological and subjective sexual response, sexual desire, and salivary steroid hormones in healthy premenopausal women. J Sex Med 2009;6:739-751.
\end{abstract}

Key Words. Testosterone; Estradiol; Cortisol; Sexual Arousal; Sexual Desire; Women; Relationships

\section{Introduction}

I n humans, steroid hormones are critically involved in fertility and reproduction, but associations with sexual behavior and function are less clear. The majority of work has been conducted with men or clinical populations of women, so our understanding of how sexuality and hormones may be associated in sexually healthy women is limited by this lack of empirical research. As a secondary effect of this, our potential understandings are limited by our inability to predict how generalizable findings from clinical populations are to healthy populations. 
Researchers examining sexuality and hormones have focused largely on the hypothalamicpituitary-gonadal and hypothalamic-pituitaryadrenal (HPA) axes, which are associated with estrogens (e.g., estradiol [E]), androgens (e.g., testosterone [T]), and cortisol (C), among other hormones, and on this article we focus on T, E, and $\mathrm{C}$, among other hormones. Though questions are often predicated upon examining how hormones influence behavior, behavioral contexts also influence hormones [1].

Evidence does provide some support for hormonal influences on certain sexual parameters, mostly from clinical populations. In women, estrogens appear to facilitate vaginal lubrication and vasocongestion [2]. Additionally, androgen administration increases some sexual parameters including desire in postmenopausal women [3], though this evidence is inconsistent and still controversial $[4,5]$. One study with a nonclinical sample of women found that $\mathrm{T}$ administration and repeated exposure to erotic stimuli led to increased sexual arousal and "sexual lust" after a time delay [6], and a follow-up found an increase in genital vasocongestion (an indirect measure of arousal) but not subjective arousal after $\mathrm{T}$ and exposure to one episode of visual erotic stimuli [7]. In contrast to $\mathrm{E}$ and $\mathrm{T}$, little research has focused on how $\mathrm{C}$ might be causally related to sexuality in women.

Evidence also supports the "reverse relationship," whereby sexual activity influences hormones. For example, intercourse and physical intimacy have been shown to increase women's T [8]. Another study [9] showed in a very small sample of four women that $\mathrm{T}$ significantly increased following sexual activity. An additional study [10] found that viewing erotic stimuli and masturbating until orgasm were associated with increased plasma $\mathrm{T}$ and luteinizing hormone (the pituitary hormone that signals the gonads to release $\mathrm{T}$ ), but had no significant effect on $\mathrm{C}$ or $\mathrm{E}$.

Others have examined how viewing erotic material influences hormones in a laboratory setting. In a small study [11] of 13 women, researchers found no association between sexual arousal and endocrine (e.g., repeated sampling of plasma $\mathrm{T}, \mathrm{C}$ ) responses to viewing erotic stimuli. They did not assess, however, whether baseline hormones predicted subsequent arousal, or whether hormones changed from pre- to postviewing. In another small study of nine women [12], the authors found that viewing erotica did not affect C. Hamilton et al. [13] found that a small sample of nine women whose $\mathrm{C}$ increased after viewing erotica had lower arousal, desire, and satisfaction scores as measured by the Female Sexual Function Index (FSFI [14]) than 20 women whose $\mathrm{C}$ decreased. Thus, evidence is somewhat mixed about the effects of viewing erotica, which may stem from viewing erotica in a laboratory environment with no physical sexual activity in accompaniment, or from methodological issues, e.g., many of these studies included women who were using hormonal contraceptives (which alter endogenous endocrinology), approaching menopause, using medications that affect hormones, etc.

The study of sexual arousal in women generally focuses on genital and subjective arousal in response to erotic stimuli, which typically show low or no correlations in premenopausal women [15]. Genital sexual arousal is usually measured using the vaginal photoplethysmograph, which measures vaginal vasocongestion by vaginal pulse amplitude (VPA). Subjective sexual arousal is measured via self-report paper-and-pencil questionnaires [16]. One might expect steroids to be responsive to and/or influencing genital and/or subjective arousal. For example, as androgen receptors are located widely throughout genital and neural tissues in women (as in men), T may be directly involved in genital and subjective arousal. And, Tuiten et al. [6,7] have found that $T$ administration can increase genital sexual arousal. Similarly, the association between $\mathrm{E}$ and both vaginal vasocongestion and lubrication [17] supports the possibility that there could be associations between genital arousal and E. Arousal generally involves the HPA axis and $\mathrm{C}$, so there may be the possibility that $\mathrm{C}$ could be directly involved with genital arousal, though research has generally produced null or mixed associations, depending on the baseline sexual response characteristics of the sample [13].

Research on associations between sexual arousal and hormones has mostly been conducted using plasma measures of hormones, often using indwelling catheters to repeatedly sample plasma. This method has several advantages, including real-time and repeated hormone sampling. It also has several drawbacks, including the invasiveness of serum sampling and its possible dampening effects on sexuality and arousal, as well as the unknown effects of blood withdrawal on physiological processes of arousal. For example, blood loss could trigger very specific patterns of physiological response that might include a down-regulation of gonadal steroid release and an up-regulation of HPA axis hormones, whereas the body deals with the "stressor" of venipuncture and a sudden drop in 
blood pressure. Salivary measures are newer but widely used and well-validated. Salivary T, for example, correlates well with free serum $\mathrm{T}$ [18-21] or total serum $\mathrm{T}[19,22]$, though there are some conflicting results [21] that raise the possibility that the use of salivary $\mathrm{T}$ in tests of hormone-behavior relationships may lead to an underestimation of effects in women that can be ameliorated with the use of larger samples of women [22].

Salivary sampling has some drawbacks as well, including the inability to continually sample saliva, but has several advantages over serum collection. Salivary hormones represent the "bioavailable" fraction, i.e., the portion that is un- or weakly bound to albumin and able to travel to receptors in the body [23]. In addition, though spitting is not generally sexual, it is unlikely to trigger the undesirable physiological reactions to blood draws, is more practical, and is less invasive overall. Further, studies of social neuroendocrinology (i.e., associations between social behavioral contexts and hormones) have more consistently found significant behavior-hormone associations with salivary sampling than plasma, further supporting the utility of saliva in behavioral and psychological studies [1]. Specifically, most studies fail to find a correlation between serum T and women's sexual desire [24], whereas some studies have found correlations using salivary $\mathrm{T}$ [8].

In the present study, we examined how viewing erotic stimuli may be associated with bidirectional links between physiological sexual arousal and steroid hormones (T, C, and E). We had four main questions that remain relatively unaddressed in the literature: (i) does viewing erotic stimuli alter T, C, and $\mathrm{E}$ in healthy premenopausal women?; (ii) do baseline levels of T, C, or E affect levels of and latency to physiological sexual arousal? (iii) do changes in hormones during viewing an erotic film parallel changes in physiological and/or subjective sexual arousal?; and (iv) how might salivary E, $\mathrm{C}$, and $\mathrm{T}$ be associated with sexual desire? We addressed these questions using salivary sampling, which may be a more appropriate medium for behavioral studies of hormone-sexuality associations, and provide one of the first studies of sexual arousal, sexual desire, and salivary $\mathrm{T}, \mathrm{E}$, and $\mathrm{C}$.

\section{Methods}

\section{Participants}

Participants were 40 women (mean age 28.18 years; standard deviation 4.52 years) recruited from a metropolitan city in the Pacific Northwest via advertisements and flyers posted on local LISTSERVS and websites. The advertisements made the sexual nature of the study clear, and we excluded potential participants based on hormonal contraceptive use. Participants were diverse by self-reported ethnicity/race, length of time in Canada, sexual orientations, occupations, contraceptives/sexually transmitted infection prevention methods, lifetime number of sexual partners, and relationship status (see Table 1). Three women had children, and participants were highly educated. Women who engaged in solitary sexual activity $(90 \%)$ reported doing so once/month (15\%), $2-3 /$ month $(12.5 \%)$, once/week $(17.5 \%)$, $2-4 /$ week (42.5\%), and once/day (2.5\%). Only one woman reported sexual difficulties (i.e., "healing from sexual assault") for which she had received treatment; no other woman reported sexual difficulties or treatment.

\section{Main Outcome Measures}

The main outcome measures of this study were: change in genital sexual arousal (VPA), change in subjective sexual arousal, sexual desire (via the Sexual Desire Inventory [SDI] and FSFI scales), as well as $\mathrm{T}, \mathrm{C}$, and $\mathrm{E}$ at time 1, time 2, and the percent change in these hormones over the viewing of the erotic film.

A questionnaire battery was administered prior to the psychophysiological testing.

\section{Health and Demographics Questionnaire}

A questionnaire developed for this study assessed a variety of health and demographic variables, to address information about participants and potential confounds with the endocrine and/or sexual measures.

\section{FSFI [14]}

A validated measure of sexual desire, orgasm, lubrication, pain, and satisfaction was administered. Because of some conceptual and statistical problems identified by Meyer-Bahlburg and Dolezal [25], adjustments were made to the scoring of the FSFI such that any woman who had not engaged in sexual activity over the preceding 4 weeks was excluded from analyses of all FSFI subscales except sexual desire.

\section{Short Form-36 Quality of Life Questionnaire (SF-36 [26])}

This is considered a gold-standard measure of functional health status and quality of life. We computed physical component and mental compo- 
Table 1 Self-reported sample characteristics (frequency and percent) for ethnicity/race, length of time in Canada, occupations, lifetime number of sexual partners, sexual orientation, relationship status (more than one category could be checked here), and contraceptive/STI prevention methods

\begin{tabular}{|c|c|c|}
\hline Measure & $\mathrm{N}$ & Percentage \\
\hline \multicolumn{3}{|l|}{ Ethnicity/race } \\
\hline Asian & 12 & $30 \%$ \\
\hline European/Canadian & 24 & $60 \%$ \\
\hline First nations/European & 2 & $5 \%$ \\
\hline Mixed heritage & 2 & $5 \%$ \\
\hline \multicolumn{3}{|l|}{ Length of time in Canada } \\
\hline Entire life & 30 & $75 \%$ \\
\hline Less than 1 year & 2 & $5 \%$ \\
\hline $1-5$ years & 2 & $5 \%$ \\
\hline $5-10$ years & 1 & $2.5 \%$ \\
\hline $10-20$ years & 3 & $7.5 \%$ \\
\hline 20-30 years & 2 & $5 \%$ \\
\hline \multicolumn{3}{|l|}{ Occupation } \\
\hline Administrative staff & 5 & $12.5 \%$ \\
\hline Nurse & 4 & $10 \%$ \\
\hline Research & 5 & $12.5 \%$ \\
\hline Retail/service & 3 & $7.5 \%$ \\
\hline Student/graduate student & 19 & $47.5 \%$ \\
\hline $\begin{array}{l}\text { Database support analyst, educator, environmental technician, graphic artist, holistic practitioner, } \\
\text { IT analyst, medical/social worker, recruiter, singer, site administrator, special needs childcare, } \\
\text { X-ray technologist, and youth worker }\end{array}$ & 1 each (13 total) & $32.5 \%$ \\
\hline No response & 6 & $15 \%$ \\
\hline \multicolumn{3}{|l|}{ Lifetime sexual partners } \\
\hline 0 & 1 & $2.5 \%$ \\
\hline $2-4$ & 7 & $17.5 \%$ \\
\hline $5-10$ & 10 & $25 \%$ \\
\hline $11-20$ & 14 & $35 \%$ \\
\hline $21-30$ & 3 & $7.5 \%$ \\
\hline $30+$ & 4 & $10 \%$ \\
\hline \multicolumn{3}{|l|}{ Sexual orientation } \\
\hline Bisexual & 4 & $10 \%$ \\
\hline Bisexual/heterosexual & 2 & $5 \%$ \\
\hline Heterosexual & 32 & $80 \%$ \\
\hline No response & 2 & $5 \%$ \\
\hline \multicolumn{3}{|l|}{ Relationship status } \\
\hline Single & 8 & $20 \%$ \\
\hline Dating one person & 6 & $15 \%$ \\
\hline Dating more than one person & 5 & $12.5 \%$ \\
\hline Long-term committed relationship $<12$ months & 6 & $15 \%$ \\
\hline Long-term committed relationship >12 months & 8 & $20 \%$ \\
\hline Married/common-law & 7 & $17.5 \%$ \\
\hline Divorced/separated & 3 & $7.5 \%$ \\
\hline \multicolumn{3}{|l|}{ Contraceptive/STI Prevention Methods } \\
\hline Condoms & 17 & $42.5 \%$ \\
\hline Condom + diaphragm & 1 & $2.5 \%$ \\
\hline Condom + cycle monitoring & 3 & $7.5 \%$ \\
\hline Condom + spermicide & 2 & $5 \%$ \\
\hline Condom + sponge & 1 & $2.5 \%$ \\
\hline Condom + withdrawal & 1 & $2.5 \%$ \\
\hline Condom + IUD (intrauterine device) & 2 & $5 \%$ \\
\hline IUD & 2 & $5 \%$ \\
\hline Tubal ligation & 1 & $2.5 \%$ \\
\hline Vasectomy: "plus monogamy and regular testing" & 1 & $2.5 \%$ \\
\hline Withdrawal (one $P$ noted "fully STD tested \& monogamous") & 2 & $5 \%$ \\
\hline No response/not currently sexually active & 7 & $17.5 \%$ \\
\hline
\end{tabular}

Some variables reflect authors' post hoc categorizations. 
nent subscores - the latter of which was used as a measure of quality of life.

\section{SDI [27]}

This was administered as a specific measure of sexual desire that has been validated with nonclinical populations of healthy women and men. It produces a total SDI score as well as dyadic and solitary SDI subscores.

\section{Detailed Assessment of Sexual Arousal (DASA [28])}

An unpublished questionnaire that has been found to significantly differentiate aspects of sexual arousal in women was administered. Subscales include "mental excitement," "genital tingling/ throbbing," and "pleasant genital sensations."

\section{The Film Scale (FS [29])}

This was administered before and after psychophysiological measurement and included subscales focused on: perception of genital sexual arousal; psychological sexual arousal; autonomic arousal; anxiety; positive affect; and negative affect. Items were rated on a 7-point Likert scale from 1 ("not at all") to 7 ("intensely").

\section{Psychophysiological Recording}

Genital arousal was measured using the vaginal photoplethysmograph, and the VPA signal was the primary end point, as it is a sensitive and specific measure of genital arousal [30]. VPA was monitored during the film and recorded on an HP Compaq nc8000 Pentium Laptop using AcqKnowledge 3.8.1 software (BIOPAC Systems Inc., Santa Barbara, CA, USA) and a data acquisition unit model MP150WSW (BIOPAC Systems Inc.) for analog/digital conversion. We used a sampling rate of 200 samples/second. The signal was bandpass filtered $(0.5-30 \mathrm{~Hz})$. We used one vaginal probe (Behavioral Technology Inc., Salt Lake City, UT, USA), and data were analyzed in 30-second segments, then averaged over the neutral and erotic segments separately, resulting in two data points per subject per session. Artifact detection following visual inspection of the data permitted the smoothing of artifacts. The vaginal probe was sterilized in a solution of Cidex OPA (orthophthalaldehyde $0.55 \%$ ), a high level disinfectant Advanced Sterilization Products (Irvine, CA, USA), immediately following each session.

Films

Participants viewed a 3-minute neutral film (either a documentary about lei making or a travelog about
Hawaii) followed by an 8-minute erotic film (a female-directed film consisting of heterosexual manual genital stimulation, oral sex, anal sex, and intercourse). Participants watched one of two film sets, each containing a neutral and erotic segment, and previous research has validated the similarity of women's arousal to and ratings of these films [31].

\section{Saliva Samples and Hormone Assays}

Participants provided two saliva samples, from which T, E, and $\mathrm{C}$ were measured. Participants chewed sugar-free Trident gum Cadbury Adams USA LLC (Parsippany, NJ, USA), and saliva was collected via passive drool into $17-\mathrm{mL}$ test tubes. Tubes were then frozen until assay. Samples were assayed at the Core Biomarkers Lab at Yerkes Primate Research Center and Emory University via radioimmunoassay. Samples were assayed for C in two batches (August 2007 and May 2008); the assay range was $0.025-10 \mathrm{ug} / \mathrm{dL}$, the intraassay coefficients of variation were $8.7 \%$ and $5.2 \%$, and the interassay coefficients of variation were $2.91 \%$ at $0.26 \mathrm{ug} / \mathrm{dL}$ and $4.39 \%$ at $1.94 \mathrm{ug} /$ $\mathrm{dL}$. Samples were assayed for $\mathrm{E}$ in one batch (June 2008); the assay range was $1-32 \mathrm{pg} / \mathrm{mL}$ at a $100-\mathrm{uL}$ dose, the intra-assay coefficients of variation $13.2 \%$ at $11.72 \mathrm{pg} / \mathrm{mL}, 8.7 \%$ at $29.45 \mathrm{pg} /$ $\mathrm{mL}$, and $15.0 \%$ at $7.67 \mathrm{pg} / \mathrm{mL}$, and the interassay coefficients of variation were $14.06 \%$ at $7.88 \mathrm{pg} / \mathrm{mL}$ and $13.81 \%$ at $23.95 \mathrm{pg} / \mathrm{mL}$. Samples were assayed for $\mathrm{T}$ in two batches (August 2007 and April 2008); the assay range was $2-500 \mathrm{pg} / \mathrm{mL}$ at a $200-\mathrm{uL}$ sample, the inter-assy coefficients of variation were $19.16 \%$ at $5.03 \mathrm{pg} /$ $\mathrm{mL}, 15.08 \%$ at $170.81 \mathrm{pg} / \mathrm{mL}$, and $16.40 \%$ at $25.31 \mathrm{pg} / \mathrm{mL}$, and the intra-assay coefficients of variation were $3.41 \%$ at $26.89 \mathrm{pg} / \mathrm{mL}$.

\section{Procedure}

This research was approved by the research ethics board of the university and hospital where this research took place. As noted earlier, participants were recruited via advertisements that clearly noted the sexual nature of the study. Participants were instructed to contact the number provided on the advertisement, and upon doing so went through a phone screening process with one of two trained female research assistants. Participants were screened out for sexual dysfunction, signs of early menopause, age above 45 years, antidepressant use, smoking, use of medications that affect hormones (including hormonal therapy), pregnancy/lactation, use of hormonal contraceptives, and medical/endocrinological conditions that 
affect hormones. To be eligible for participation, women had to have previously experienced sexual intercourse, masturbation, and/or tampon use (to ensure comfort and ability to use the vaginal probe). Testing occurred between 11:00 and 18:00 h to avoid the notably high and rapidly declining steroid levels associated with waking and/or the morning $[18,32]$ and to meet the needs of participants' schedules. However, because of scheduling conflicts, five participants were tested prior to $11: 00 \mathrm{~h}$ (09:30, 10:10, 10:15, 10:40 ×2). Participants were tested between 2007 and 2008 in a hospital research room that contained a comfortable reclining chair, two bookcases, large screen TV, an intercom, and a sink with small cupboard unit. A thin blanket was placed over the seating area of the chair.

Participants were tested by female researchers, and provided written consent after viewing the lab apparatus and asking questions about the lab procedure. Participants completed the questionnaire battery and provided the first saliva sample (i.e., T1, E1, and C1). Participants were provided with further instructions about the photoplethysmograph and the remainder of the study. The researcher left the room; participants inserted the probe and informed the researcher of their readiness via intercom. The audio component was delivered via wireless headphones. The film sequence started with 1 minute of the word "Relax" on the screen; participants then watched the 3 -minute neutral film, and then viewed the 8-minute erotic film. Participants then completed a second film scale and provided the second saliva sample (T2, E2, C2). As participants responded to the questionnaire battery and got comfortable in the testing room at different rates, there was some variability between the two saliva samples (mean 51 minutes; range 25-90 minutes). Participants were then debriefed and provided with a $\$ 20$ reimbursement for their time. Saliva samples were frozen after collection until assay.

Participants were tested at all phases in their menstrual cycles except for menstruation. As suggested by previous research [33], cycle phase does not need to be controlled for in studies with $\mathrm{T}$ unless cycle phase is of particular interest. E changes rapidly over the cycle, with a high but brief preovulatory peak and a gradual increase during the first half, and gradual decline during the second half of the luteal phase. Changes in $\mathrm{C}$ over the menstrual cycle have been less well characterized; research indicates that variation in salivary $\mathrm{C}$ over the menstrual cycle is small, variable, or nonsignificant [34-36].

\section{Results}

Data were analyzed using SPSS, version 15 (SPSS Inc., Chicago, IL, USA). Post hoc analyses were conducted using the least significant difference statistic.

We conducted analyses on changes in genital arousal and hormones using absolute and relative changes. We reported our analyses on absolute changes to aid in comparing results from this study to others, as researchers in the field generally analyze these values. We additionally reported our analyses on relative changes because both genital arousal and hormones do not have absolute metrics. Individual starting points and capacity for change vary widely, and percent change scores thus provided a measure of each individual's relative change. Analyses with percent changes take into account small absolute changes that may be large relative to individual starting points in ways that absolute values do not. Previous studies $[8,37]$ have shown the utility of analyzing percent changes as more sensitive measures of changes in the face of large individual variability.

The percent change in each hormone (i.e., T\%, $\mathrm{C} \%, \mathrm{E} 2 \%)$ was calculated as the posttest value minus the pretest value, divided by the pretest value. We conducted analyses with both times 1 and 2 (corresponding to pre- and post-viewing times, respectively), and thus levels potentially modulated by the erotic film. Women were divided by median split into high and low T1, $\mathrm{E} 1$, and $\mathrm{C} 1$ groups to facilitate dichotomous comparisons.

The percent change in genital arousal was calculated as: $\mathrm{VPA} \%=\mathrm{VPA}_{\text {erotic }}-\mathrm{VPA}_{\text {neutral }}$, all divided by $\mathrm{VPA}_{\text {neutral }}$. We also identified the latency to maximum VPA during the erotic film; this consisted of the earliest peak-to-trough occurrence of maximum VPA, within one standard deviation of that individual's arousal scores. We also calculated a within-subject relative genital arousal score to the erotic film: this consisted of the highest peak of $\mathrm{VPA}_{\text {erotic }}$ minus the lowest $\mathrm{VPA}_{\text {erotic }}$. Self-report arousal analyses were conducted using difference scores that reflected the post-viewing score minus the previewing score on each of the six FS domains.

Outliers (over 3SD from the mean) on each measure were removed only from related analyses. Two women using medications that have known or potential effects on $\mathrm{T}, \mathrm{C}$, or $\mathrm{E} 2$ were removed from hormone analyses. T1 was unmeasurable in two women, and these women's T\% could not be 
Table 2 Means and standard deviations on the Female Sexual Function Index (FSFI), Detailed Assessment of Real-Life Sexual Arousal (DASA), and Rand 36-Item Health Survey (SF36)

\begin{tabular}{lrr}
\hline Measure & Mean & $\begin{array}{c}\text { Standard } \\
\text { deviation }\end{array}$ \\
\hline FSFI & & \\
Desire & 4.22 & 0.85 \\
Arousal & 5.26 & 0.58 \\
Lubrication & 5.53 & 0.58 \\
Orgasm & 5.11 & 0.89 \\
Satisfaction & 4.82 & 1.04 \\
Pain (high scores = lower pain) & 5.44 & 0.93 \\
Total & 29.98 & 3.23 \\
DASA & & \\
Mentally excited & 5.29 & 0.74 \\
Genital tingling & 5.30 & 0.76 \\
Genital wetness & 5.90 & 0.77 \\
SF36 & & \\
Physical functioning & 92.63 & 13.25 \\
Role limitations caused by physical health & 86.04 & 30.04 \\
Role limitations caused by emotional & 83.04 & 28.48 \\
$\quad$ problems & & \\
Energy/fatigue & 46.95 & 10.21 \\
Emotional well-being & 36.99 & 7.97 \\
Social functioning & 69.63 & 17.37 \\
Pain & 85.56 & 14.14 \\
General health & 75.53 & 19.15 \\
\hline$\quad$ & \\
\hline
\end{tabular}

calculated; E1 could not be measured in four women, E2 could not be measured in two women (one of whose E1 could not be measured), thus $\mathrm{E} \%$ of five women could not be calculated. There were two outliers in VPA, five T1 outliers, five T2 outliers (three of whom were already T1 outliers), three $\mathrm{C} 1$ outliers, one $\mathrm{C} 2$ outlier, two $\mathrm{C} \%$ outliers, two E2 outliers (one of whom was an outlier on T1 and one of whom was an outlier on T2), and one $\mathrm{E} \%$ outlier.

As age, body mass index (BMI), and time of sampling may be confounded with the measures, we conducted analyses of variance (ANOvAs) that controlled for these factors when they accounted for significant portions of variance, and included all three factors in partial correlations.

\section{Sexual Characteristics of the Sample}

Scores on the FSFI, DASA, and SF36 are presented in Table 2. All domains of the FSFI were in the range reported for sexually healthy premenopausal women [38]. Scores on the DASA suggested high-moderate levels of self-reported sexual arousal. Scores on the SF36 suggested a mentally and physically healthy sample.

\section{Physiological and Subjective Arousal}

VPA\% was significantly greater than zero, $t(37)=6.50, P<0.001$, and erotic VPA was significantly higher than neutral VPA, $t(37)=7.15$, $P<0.001$ (Table 3).
Table 3 Genital sexual arousal (vaginal pulse amplitude [VPA]) and mean self-report measures of arousal and affect before and after viewing an erotic film (scale range 1-7)

\begin{tabular}{|c|c|c|c|c|}
\hline \multirow[b]{2}{*}{ Measures } & \multicolumn{2}{|c|}{ Neutral stimulus } & \multicolumn{2}{|c|}{ Erotic stimulus } \\
\hline & Mean & $\begin{array}{l}\text { Standard } \\
\text { deviation }\end{array}$ & Mean & $\begin{array}{l}\text { Standard } \\
\text { deviation }\end{array}$ \\
\hline VPA (mV) & 0.0452 & 0.0392 & 0.0882 & 0.0965 \\
\hline $\begin{array}{l}\text { Perception of genital } \\
\text { arousal }\end{array}$ & 1.97 & 1.30 & 3.92 & 1.23 \\
\hline $\begin{array}{l}\text { Subjective sexual } \\
\text { arousal }\end{array}$ & 1.97 & 0.95 & 3.72 & 1.50 \\
\hline Autonomic arousal & 1.86 & 1.05 & 3.25 & 1.03 \\
\hline Positive affect & 2.22 & 1.26 & 3.69 & 1.16 \\
\hline Negative affect & 1.30 & 0.52 & 1.33 & 0.33 \\
\hline Anxiety & 1.70 & 1.11 & 1.30 & 0.61 \\
\hline
\end{tabular}

We conducted a repeated measures anova to examine changes in self-reported subjective arousal from pre- to post-viewing, and there was a significant effect, multivariate $F(6,34)=21.93$, $P<0.001$, with all subscale scores showing a significant change except for negative arousal, $F(1,39)=0.15, \quad P=0.738$. Overall arousal increased significantly, $F(1,39)=71.89, P<0.001$, as did autonomic arousal, $F(1,39)=70.43, P<$ 0.001 , genital arousal, $F(1,39)=71.88, P<0.001$, and positive arousal $F(1,39)=43.96, P<0.001$. Anxious arousal decreased significantly, $F(1,39)$ $=4.66, P<0.037$ (see Table 3 ).

VPA\% differed significantly by relationship status in an ANOva controlling for age and BMI, $F(2,33)=5.74, P=0.007$ (see Figure 1). Dating women had significantly higher VPA\% than

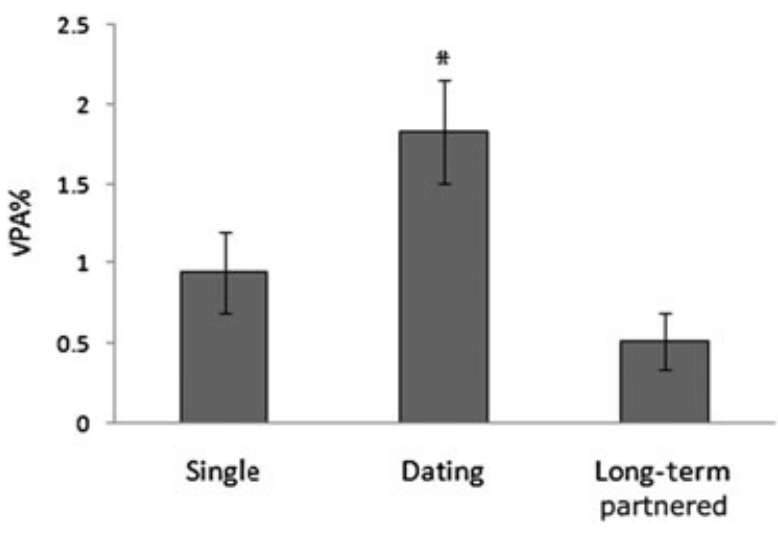

Figure 1 Percent change in vaginal pulse amplitude (VPA\%) by relationship status in single $(\mathrm{N}=9)$, dating $(\mathrm{N}=8)$, and long-term partnered women $(\mathrm{N}=21)$, with age and body mass index as covariates. The asterisk indicates a significant difference from the other relationship statuses at $P<0.05$. 


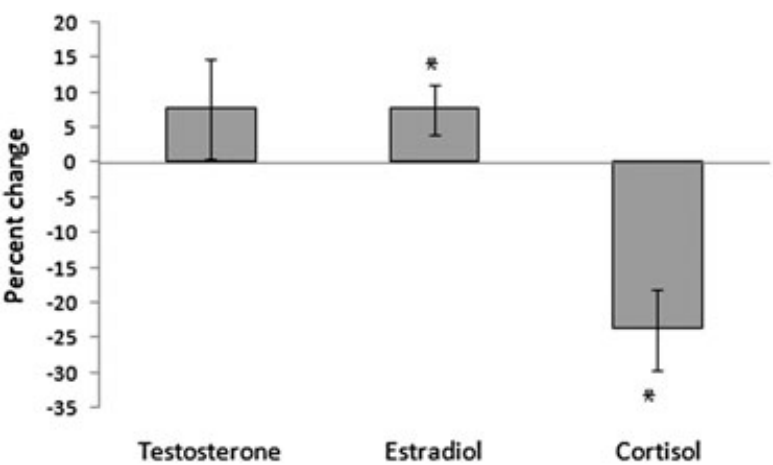

Figure 2 Relative changes in testosterone $(\mathrm{N}=30)$, cortisol $(\mathrm{N}=32)$, and estradiol $(\mathrm{N}=31)$ from Time 1 to Time 2 with standard error bars; percent changes calculated as hormones at time 2 minus time 1 , divided by time 1 . Asterisks indicate a significant difference from zero at $P<0.05$.

single women, $P=0.045$, and long-term partnered women, $P=0.002$. There were no significant differences in self-reported sexual arousal by relationship status.

VPA\% was not significantly correlated with any of the DASA subscales.

\section{Hormonal Changes Over Viewing Time}

We conducted a repeated measures anova to compare the percent changes in $\mathrm{C}, \mathrm{T}$, and $\mathrm{E}$, and this was significant $-F(2,40)=12.77, P<0.001$. $\mathrm{T} \%$ was significantly higher than $\mathrm{C} \%, P<0.001$, but was not significantly different from $\mathrm{E} \%$, $P=0.519$. E\% was significantly higher than $\mathrm{C} \%$, $P<0.001$ (see Figure 2). The percent change in hormones did not differ significantly by relationship status.

\section{Testosterone}

$\mathrm{T} 1$ and $\mathrm{T} 2$ were not significantly different (see Figure 3), paired $t(29)=0.68, P=0.501$, and T\% was not significantly different than zero (see Figure 2), $t(29)=1.08, P=0.289$.

There were no significant correlations between T and VPA\% (see Table 4 for partial correlations), magnitude of the relative increase in VPA during the erotic condition (see Table 4), or latency to maximum VPA during the erotic condition.

There was a trend for T1 to be correlated with the increase in autonomic arousal, $r(30)=-0.33$, $P=0.065$. Controlling for factors allowed this correlation to reach significance, $r(26)=0.38$, $P=0.046$, but controlling for multiple contrasts made this correlation nonsignificant. None of the other $\mathrm{T}$ measures were significantly correlated with the self-report arousal scores.

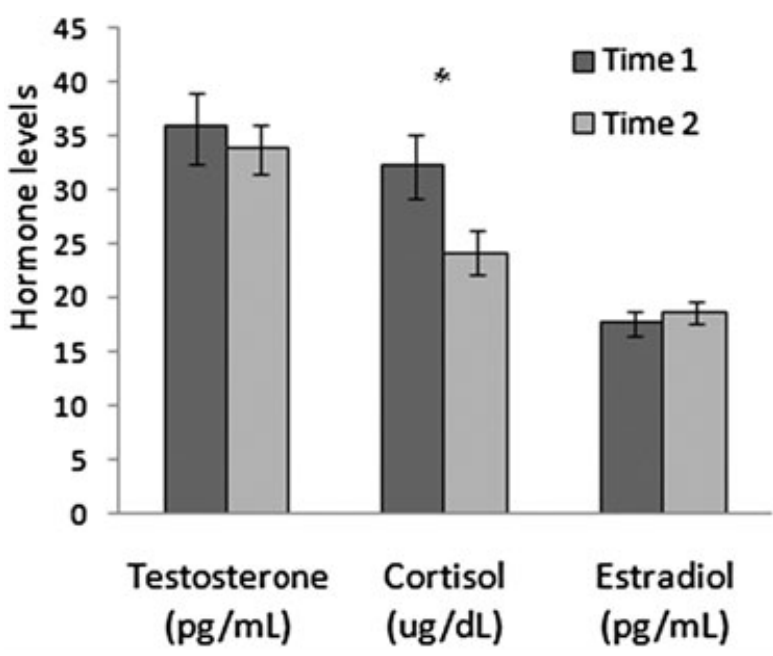

Figure 3 Testosterone $(\mathrm{N}=30)$, cortisol $(\mathrm{N}=34)$, and estradiol $(\mathrm{N}=32)$ at times 1 and 2, with standard error bars. The asterisk indicates a significant difference in cortisol between Times 1 and 2 .

There were no significant partial correlations between the DASA subscales and T1, or T\%. T2, however, was significantly correlated with the DASA subscale, Mental sexual excitement, partial $r(26)=0.55, \quad P=0.003$; the Genital Wetness DASA subscale, partial $r(26)=0.50, P=0.006$; and nearly so with the Genital Tingling DASA subscale, partial $r(26)=0.34, P=0.080$. These remained significant correlations after controlling for multiple comparisons, excepting genital tingling. There was a trend for T1 to be correlated with the orgasm FSFI subscale, partial $r(21)=$ $0.39, P=0.066$, which was nonsignificant after controlling for multiple comparisons. There were no other correlations between the FSFI subscales and $\mathrm{T} 1, \mathrm{~T} 2$, or $\mathrm{T} \%$. There were no significant

Table 4 Partial correlations between percent change in vaginal pulse amplitude (VPA\%), and magnitude of the relative increase in VPA (maximum VPA) and testosterone, cortisol, and estradiol (controlling for age, body mass index, and time of sample)

\begin{tabular}{lll}
\hline Hormones & VPA\% & Maximum VPA \\
\hline Testosterone 1 & $r(25)=0.26, \mathrm{~ns}$ & $r(25)=-0.08, \mathrm{~ns}$ \\
Testosterone 2 & $r(26)=-0.23, \mathrm{~ns}$ & $r(23)=-0.00, \mathrm{~ns}$ \\
T\% & $r(23)=-0.00, \mathrm{~ns}$ & $r(23)=-0.19, \mathrm{~ns}$ \\
Estradiol 1 & $r(28)=0.01, \mathrm{~ns}$ & $r(28)=0.29, \mathrm{~ns}$ \\
Estradiol 2 & $r(28)=0.00, \mathrm{~ns}$ & $r(28)=-0.31, P=0.096$ \\
E\% & $r(26)=0.22, \mathrm{~ns}$ & $r(26)=0.14, \mathrm{~ns}$ \\
Cortisol 1 & $r(28)=0.18, \mathrm{~ns}$ & $r(28)=0.21, \mathrm{~ns}$ \\
Cortisol 2 & $r(30)=0.36, P=0.046^{*}$ & $r(30)=0.13, \mathrm{~ns}$ \\
C\% & $r(26)=-0.15, \mathrm{~ns}$ & $r(26)=-0.05, \mathrm{~ns}$ \\
\hline
\end{tabular}

${ }^{*} P<0.05 ;$ ns $=$ nonsignificant. 
differences between $\mathrm{T}$ responders and nonresponders in their FSFI subscale scores, $F(6,23)=$ $0.51, P=0.795$.

\section{Estradiol}

E1 and E2 did not differ significantly (see Figure 3), paired $t(31)=-1.58, P=0.124$, but $\mathrm{E} \%$ was significantly greater than zero (see Figure 2), $t(30)=2.12, P=0.043$. There was no significant correlation between the amount of time that passed between samples and the relative increase in $\mathrm{E}, r(28)=-0.13, P=0.484$.

The magnitude of the relative increase in VPA was significantly correlated with $\mathrm{E} 1, r(31)=0.42$, $P=0.014$, and E2, $r(31)=0.40, P=0.021$. Controlling for factors in a partial correlation reduced the E2 correlation to a trend, partial $r(28)=0.31$, $P=0.096$, and the E1 correlation to nonsignificance, $r(28)=0.29, P>0.05$. There were no other significant correlations between E and VPA\% (see Table 4 for partial correlations), the magnitude of the relative increase in VPA during the erotic condition (see Table 4), or the latency to maximum VPA during the erotic condition.

None of the E measures were significantly correlated with the self-reported arousal scores.

There were no significant partial correlations between the DASA or FSFI subscales and E1, E2, or $\mathrm{E} \%$, except for: one significant negative partial correlation between $\mathrm{E} \%$ and the genital wetness DASA subscale, partial $r(26)=-0.44, P=0.019$; significant partial correlations between E2 and the orgasm FSFI subscale, partial $r(22)=0.47$, $P=0.019$; and the pain FSFI subscale, partial $r(22)=-0.41, P=0.05$. Controlling for multiple comparisons made these correlations nonsignificant. There were no significant differences between $\mathrm{E}$ responders and nonresponders in FSFI scores, $F(6,20)=1.50, P=0.229$.

\section{Cortisol}

$\mathrm{C} 1$ and C2 differed significantly (see Figure 3), paired $t(33)=3.40, P=0.002$, and $\mathrm{C} \%$ was significantly lower than zero (see Figure 2$), t(31)=$ $-4.15, P<0.001$.

There was a trend for a correlation between $\mathrm{C} 2$ and $\mathrm{VPA} \%, r(33)=0.29, P=0.088$, which was significant after controlling for factors, partial $r(30)=0.36, P=0.046$, but not after multiple comparisons. There were no other significant correlations between $\mathrm{C}$ and VPA\% (see Table 4 for partial correlations), the magnitude of the relative increase in VPA during the erotic condition (see Table 4), or the latency to maximum VPA during the erotic condition.
Neither C1, C2, nor C\% was significantly correlated with the self-report arousal scores. There were no significant differences between $\mathrm{C}$ responders and nonresponders in FSFI subscale scores, $F(6,25)=0.40, P=0.875$.

There were no significant partial correlations between the DASA subscales and $\mathrm{C} 1$ or $\mathrm{C} \%$. There was a trend for a significant correlation between $\mathrm{C} 2$ and the genital wetness DASA subscale, partial $r(29)=0.35, P=0.053$. There were no significant partial correlations between the FSFI subscales and $\mathrm{C} 2$ or $\mathrm{C} \%$. There was a significant correlation between $\mathrm{C} 1$ and the satisfaction FSFI subscale, partial $r(25)=-0.45$, $P=0.019$, and trends with the arousal FSFI subscale, partial $r(25)=-0.37, P=0.055$, and the total FSFI score, partial $r(25)=-0.34, P=0.086$, but these were nonsignificant after controlling for multiple comparisons.

\section{Sexual Desire and Hormones \\ Testosterone}

Only T2 was significantly correlated with solitary SDI, $r(31)=0.42, P=0.015$, and controlling for factors or multiple comparisons did not change this pattern. There were no other correlations between $\mathrm{T}$ and SDI. There were no significant correlations between the $\mathrm{T}$ measures and the desire FSFI subscale.

\section{Cortisol}

There was a trend for $\mathrm{C} 1$ to be correlated with dyadic SDI, $r(33)=0.30, P=0.080$, and controlling for factors led to a trend for $\mathrm{C} 2$ to be correlated with dyadic SDI, partial $r(31)=0.33$, $P=0.059$. There was a trend for $\mathrm{C} \%$ to be correlated with total SDI, $r(30)=0.33, P=0.065$, and controlling for factors did not change this pattern, though controlling for multiple comparisons did make these nonsignificant. There were no other significant correlations between $\mathrm{C}$ and SDI. There were no significant correlations between the $\mathrm{C}$ measures and the desire FSFI subscale.

\section{Estradiol}

There were no significant correlations between any of the $\mathrm{E}$ measures and the SDI measures. There was, however, a significant correlation between the desire FSFI subscale and $\mathrm{E} \%$, partial $r(21)=0.54, P=0.007$, which was significant after controlling for multiple comparisons, and a trend for $\mathrm{E} 1$, partial $r(23)=-0.39, P=0.054$. 


\section{Discussion}

In this study, we examined how three steroid hormones ( $\mathrm{T}, \mathrm{E}$, and $\mathrm{C}$ ) might predict or be altered by sexual arousal during viewing of erotic stimuli, and also how they might be associated with sexual desire in healthy premenopausal women.

Though we expected $\mathrm{T}$ to increase with sexual arousal, instead it was $\mathrm{E}$ that significantly increased over the viewing of erotic stimuli. There were some weak indications that this increase in $\mathrm{E}$ might be associated with increased sexual arousal, but these correlations became nonsignificant when age, BMI, and sample time were controlled. Thus, $\mathrm{E}$ may increase independently of measured sexual arousal upon viewing erotic stimuli. Our analyses also showed that the increase in E could not have been caused by time, as E\% was not significantly correlated with the amount of time that had passed. In support of this interpretation, steroid levels decline over the day, so the increase in $\mathrm{E}$ displayed in our study represents a sociallystimulated change.

Previous research [8] has shown the utility of using percent changes when examining changes in hormones over time, because both hormone levels and their changes show large variability, and relative change analyses are far more sensitive in the face of this large variation than simple comparisons of average time 1 levels to average time 2 levels. Controlling for multiple comparisons left the following correlations nonsignificant, but as exploratory findings they may still be meaningful. We found that E2 was significantly correlated with FSFI orgasm subscale scores and negatively with FSFI pain scores, which could be interpreted as consistent with research showing that higher $\mathrm{E}$ is associated with more genital vasocongestion [2]. However, in mild opposition to this interpretation, $\mathrm{E} \%$ was negatively correlated with the genital wetness DASA subscale suggesting that stimulated changes in $\mathrm{E}$ are not associated with regularly higher self-reported genital wetness.

Our study is the first to examine correlations between $\mathrm{E}$ and sexual desire using the SDI scale [27] in healthy women, and our findings suggest no association. Our study is also one of the first to examine correlations between $\mathrm{E}$ and sexual desire using the FSFI desire subscale [14]. We found a significant correlation between the FSFI desire score and $\mathrm{E} \%$, and a trend for a negative correlation with $\mathrm{E} 1$. These correlations may suggest that $\mathrm{E}$ responsiveness is a more important correlate of sexual desire than baseline E, a possibility that has far-reaching ramifications for research on hormone-desire correlations. Further research is needed to confirm this, and to reconcile the null findings with the SDI scales but significant findings with the FSFI scales.

Despite our expectations, we did not find that $T$ significantly increased with viewing erotic stimuli or was associated with sexual arousal, replicating one past study [39]. Given the large variation and resultant error bars, it remains possible that an increase in $\mathrm{T}$ might be apparent with a larger sample. As well, steroids decrease with passage of time, and previous research has shown that $\mathrm{T}$ decreases significantly over shorter periods [37], so no statistical change in $\mathrm{T}$ might reflect an attenuated decrease. However, our lack of a nonerotic viewing condition (we used passage of time and percent changes relative to zero instead) renders us unable to confirm this, which is a weakness of this study. An additional limitation includes the modest sample size, despite being larger than previous studies. We also found no significant associations between $T$ and sexual arousal, except that $T 1$ was significantly correlated with self-reported autonomic sexual arousal. This is a novel finding, and is supported by research suggesting correlations between $\mathrm{T}$ administration and sexual responses to erotic stimuli [6]; however, this finding disappeared with corrections for multiple comparisons. We also found strong positive correlations between T2 and the three DASA subscales, including mental excitement, genital wetness, and genital tingling. This suggests that stimulated levels of $\mathrm{T}$ (i.e., post-viewing) are associated with women's self-reported mental and physical sexual arousal in their natural (i.e., non-laboratory) environments. We also found a significant correlation between T1 and the FSFI orgasm subscale. This is supported by previous research [8] in which women with higher $\mathrm{T}$ reported more frequent orgasms and were also more likely to experience orgasm during sexual activity.

We also found that T2 was correlated with solitary sexual desire, which suggests that stimulated $\mathrm{T}$ may be more strongly associated with desire than $T$ levels when women are not aroused. Other studies have also been suggestive of associations between $\mathrm{T}$ and solitary-but not dyadic-sexual desire [8]. In contrast to the findings with E, there were no significant correlations between the $\mathrm{T}$ measures and the FSFI desire score, suggesting along with previous evidence of SDI-T links that in healthy women the SDI may be a more useful measure. This is likely because measurement of 
desire on the FSFI is comprised of only two items (focusing on frequency and intensity of desire, undefined), whereas 15 items are used to measure sexual desire more comprehensively on the SDI.

$\mathrm{C}$ showed a significant decline during the erotic stimuli, as previous studies have shown [13]. Interestingly, we found that post-viewing $\mathrm{C}$ was significantly and positively correlated with genital arousal, suggesting that stimulated $\mathrm{C}$ may reflect physiological processes that parallel those that underlie genital arousal. As such, increased genital arousal and $\mathrm{C}$ may reflect similar underlying processes mediating arousal. In support of this interpretation, stimulated C (i.e., C2) was significantly positively correlated with the DASA genital wetness score. Our findings failed to support Hamilton et al.'s [13] recent study suggesting that women who show an increase in C differ in FSFI scores relative to women who show a decrease in $\mathrm{C}$ when viewing erotic stimuli. Our study had a similar though slightly larger sample size, but we included only women who were not using hormonal contraceptives and who were not close to menopausal age; perhaps these controls may have influenced the differential outcome, and future replication of Hamilton et al.'s finding is clearly needed. Interestingly, we also found strong negative correlations between $\mathrm{C} 1$ and the FSFI subscales of satisfaction, arousal, and total scores (though nonsignificant with multiple comparisons). This may suggest that baseline C (in contrast to stimulated C) may be in some way inhibitory or at least negatively associated with some sexual parameters.

We found no significant correlations between the FSFI desire score and the $\mathrm{C}$ measures, but our findings did suggest that $\mathrm{C}$ might be correlated with dyadic sexual desire. Though these correlations were large, they were weak statistical trends and were nonsignificant after control for multiple comparisons. Thus, this remains to be replicated, especially with larger samples, but provides some of the first research suggestive of associations between $\mathrm{C}$ and dyadic sexual desire in women. Recent research supporting this potential association has shown associations between $\mathrm{C}$ and temporary physical separation and reunion between romantic partners [40]. It is possible that $\mathrm{C}$ is more sensitive to the dyadic and interpersonal aspects of a sexual relationship than it is to the solitary component.

An interesting and unpredicted finding was that women's sexual arousal was related to their relationship status, as dating women had higher genital sexual arousal than single or partnered women. These findings could not be attributed to age, as we controlled for the effects of age in the statistical analyses. However, this pattern was restricted to genital arousal, as psychological sexual arousal showed no association with relationship status. If future research does support this unexpected finding, this is strongly suggestive that social factors need to be attended to when examining physiological responses [1], including genital sexual arousal. The dating phase of relationships may involve specific physiological changes and/or profiles, and this speculative possibility remains open to future inquiry and substantiation.

Our study provides some of the first evidence showing that $\mathrm{E}$ increases with viewing erotic stimuli in healthy premenopausal women, perhaps independently of sexual arousal. Additionally, our data are some of the first to indicate that higher stimulated $\mathrm{C}$ and genital arousal after viewing erotic stimuli are associated, and that $\mathrm{C}$ may be associated with sexual desire in healthy women. Counter to our expectations, $\mathrm{T}$ was not associated with sexual arousal via viewing erotic stimuli in any way. Our expectation that $\mathrm{T}$ might increase was based on evidence that sexual activity leads to increased salivary $\mathrm{T}$ in women [8]; however, plasma $\mathrm{T}$ has not shown increases upon viewing erotic stimuli (in small samples of women [11]). Moreover, evidence is mixed as to whether sexual desire and endogenous $\mathrm{T}$ are associated in healthy women $[8,41]$ or clinical populations of women [24]. Our findings converge with past studies, such that viewing sexual stimuli leads to divergent physiological outcomes from engaging in sexual activity in women. This provides important data suggesting that studies that address women's sexuality and hormones via measurement of sexual arousal postexposure to erotic stimuli may be tapping into different physiological processes than those that incorporate sexual activity. Additionally, our findings provide a strong imperative for conceptualizing $\mathrm{C}$ (as well as $\mathrm{E}$ and $\mathrm{T}$ ) in separate domains, i.e., baseline levels, relative changes, and stimulated levels, as evidence from this study suggest that hormones in these three "domains" can be differentially, and sometimes oppositionally, associated with some sexual parameters.

\section{Acknowledgments}

We would like to acknowledge financial support from the UBC Hampton Grant and a Student Research 
Grant to S.M. van Anders from the Society for the Scientific Study of Sexuality to fund this study.

Corresponding Author: Sari M. van Anders, Departments of Psychology and Women's Studies, Neuroscience Program University of Michigan, Ann Arbor, MI, USA. Tel: 1-734-647-6981; Fax: 1-734-763-7480; E-mail:smva@umich.edu

Conflict of Interest: None declared.

\section{Statement of Authorship}

\section{Category 1}

(a) Conception and Design

Sari M. van Anders; Lori Brotto

(b) Acquisition of Data

Sari M. van Anders; Lori Brotto; Janine Farrell; Morag Yule

(c) Analysis and Interpretation of Data

Sari M. van Anders; Lori Brotto

\section{Category 2}

(a) Drafting the Article

Sari M. van Anders; Lori Brotto

(b) Revising It for Intellectual Content

Sari M. van Anders; Lori Brotto

\section{Category 3}

(a) Final Approval of the Completed Article

Sari M. van Anders; Lori Brotto; Janine Farrell; Morag Yule

\section{References}

1 van Anders SM, Watson NV. Social neuroendocrinology: Effects of social contexts and behaviors on sex steroids in humans. Hum Nat 2006;17:212-37.

2 Sarrel PM. Effects of hormone replacement therapy on sexual psychophysiology and behavior in postmenopause. J Womens Health Gend Based Med 2000;9(suppl 1):S25-32.

3 Hubayter Z, Simon JA. Testosterone therapy for sexual dysfunction in postmenopausal women. Climacteric 2008;11:181-91.

4 Basson R. Hormones and sexuality: Current complexities and future directions. Maturitas 2007; 57:66-70.

5 Tiefer L. Omissions, biases, and nondisclosed conflicts of interest: Is there a hidden agenda in the NAMS position statement? MedGenMed 2005;7:59.

6 Tuiten A, Van Honk J, Koppeschaar H, Bernaards C, Thijssen J, Verbaten R. Time course of effects of testosterone administration on sexual arousal in women. Arch Gen Psychiatry 2000;57:149-53.

7 Tuiten A, van Honk J, Verbaten R, Laan E, Everaerd W, Stam H. Can sublingual testosterone increase subjective and physiological measures of laboratory- induced sexual arousal? Arch Gen Psychiatry 2002; 59:465-6.

8 van Anders SM, Hamilton LD, Schmidt N, Watson NV. Associations between testosterone secretion and sexual activity in women. Horm Behav 2007; 51:477-82.

9 Dabbs JM Jr, Mohammed S. Male and female salivary testosterone concentrations before and after sexual activity. Physiol Behav 1992;52:195-7.

10 Exton MS, Bindert A, Kruger T, Scheller F, Harmann U, Schedlowski M. Cardiovascular and endocrine alterations after masturbation induced orgasm in women. Psychosom Med 1999;61:280-9.

11 Heiman JL, Rowland DL, Hatch JP, Gladue BA. Psychophysiological and endocrine responses to sexual arousal in women. Arch Sex Behav 1991; 20:171-86.

12 Exton NG, Truong TC, Exton MS, Wingenfeld SA, Leygraf N, Saller B, Hartmann U, Schedlowski M. Neuroendocrine response to film-induced sexual arousal in men and women. Psychoneuroendocrinology 2000;25:187-99.

13 Hamilton LD, Rellini AH, Meston CM. Cortisol, sexual arousal, and affect in response to sexual stimuli. J Sex Med 2008;5:2111-8.

14 Rosen R, Brown C, Heiman J, Leiblum S, Meston C, Shabsigh R, Ferguson D, D'Agostino R Jr. The Female Sexual Function Index (FSFI): A multidimensional self-report instrument for the assessment of female sexual function. J Sex Marital Ther 2000;26:191-208.

15 Rosen RC, Beck JG. Patterns of sexual arousal. New York: Guilford Press; 1988.

16 Laan E, Everaerd W. Physiological measures of vaginal vasocongestion. Int J Impot Res 1998;2(10 suppl):S107-10.

17 Laan E, van Lunsen RH. Hormones and sexuality in postmenopausal women: A psychophysiological study. J Psychosom Obstet Gynaecol 1997;18:12633.

18 Khan-Dawood FS, Choe JK, Dawood MY. Salivary and plasma bound and "free" testosterone in men and women. Am J Obstet Gynecol 1984;148:441-5.

19 Granger DA, Shirtcliff EA, Booth A, Kivlighan KT, Schwartz EB. The "trouble" with salivary testosterone. Psychoneuroendocrinology 2004;29:1229-40.

20 Magrini G, Chiodoni G, Rey F, Felber JP. Further evidence for the usefulness of the salivary testosterone radioimmunoassay in the assessment of androgenicity in man in basal and stimulated conditions. Horm Res 1986;23:65-73.

21 Swinkels LM, Meulenberg PM, Ross HA, Benraad TJ. Salivary and plasma free testosterone and androstenedione levels in women using oral contraceptives containing desogestrel or levonorgestrel. Ann Clin Biochem 1988;25(4 pt):354-9.

22 Shirtcliff EA, Granger DA, Likos A. Gender differences in the validity of testosterone measured in saliva by immunoassay. Horm Behav 2002;42:62-9. 
23 Quissell DO. Steroid hormone analysis in human saliva. Ann N Y Acad Sci 1993;694:143-5.

24 Schover LR. Androgen therapy for loss of desire in women: Is the benefit worth the breast cancer risk? Fertil Steril 2008;90:129-40.

25 Meyer-Bahlburg HF, Dolezal C. The female sexual function index: A methodological critique and suggestions for improvement. J Sex Marital Ther 2007;33:217-24.

26 Ware JE Jr, Sherbourne CD. The MOS 36-item short-form health survey (SF-36). I. Conceptual framework and item selection. Med Care 1992; 30:473-83.

27 Spector IP, Carey MP, Steinberg L. The sexual desire inventory: Development, factor structure, and evidence of reliability. J Sex Marital Ther 1996;22:175-90.

28 Basson R, Brotto LA. Sexual psychophysiology and effects of sildenafil citrate in oestrogenised women with acquired genital arousal disorder and impaired orgasm: A randomized controlled trial. BJOG 2001;110:1014-24.

29 Heiman JR, Rowland DL. Affective and physiological sexual response patterns: The effects of instructions on sexually functional and dysfunctional men. J Psychosom Res 1983;27:105-16.

30 Laan E, Everaerd W, Evers A. Assessment of female sexual arousal: Response specificity and construct validity. Psychophysiology 1995;32:476-85.

31 Brotto LA, Basson R, Luria M. A mindfulness-based group psychoeducational intervention targeting sexual arousal disorder in women. J Sex Med 2008; 5:1646-59.

32 Axelsson J, Ingre $M$, Skerstedt T, Holmback U. Effects of acutely displaced sleep on testosterone. J Clin Endocrinol Metab 2005;90:4530-5.
33 Dabbs JM Jr, de La Rue D. Salivary testosterone measurements among women: Relative magnitude of circadian and menstrual cycles. Horm Res 1991;35:182-4.

34 Odber J, Cawood EH, Bancroft J. Salivary cortisol in women with and without perimenstrual mood changes. Psychosom Res 1998;45:557-68.

35 Kirschbaum C, Kudielka BM, Gaab J, Schommer NC, Hellhammer DH. Impact of gender, menstrual cycle phase, and oral contraceptives on the activity of the hypothalamus-pituitary-adrenal axis. Psychosom Med 1999;61:154-62.

36 McCormick CM, Teillon SM. Menstrual cycle variation in spatial ability: Relation to salivary cortisol levels. Horm Behav 2001;39:29-38.

37 van Anders SM, Watson NV. Ability- vs. chancedetermined competition outcomes: Effects on testosterone in humans. Physiol Behav 2007;90:634-42.

38 Wiegel M, Meston CM, Rosen R. The female sexual function index (FSFI): Cross-validation and development of clinical cutoff scores. J Sex Marital Ther 2005;31:1-20.

39 Hamilton LD, Fogle EA, Meston CM. The roles of testosterone and alpha-amylase in exercise-induced sexual arousal in women. J Sex Med 2008;5:84553

40 Diamond LM, Hicks AM, Otter-Henderson KD. Every time you go away: Changes in affect, behavior, and physiology associated with travel-related separations from romantic partners. J Pers Soc Psychol 2008;95:385-403.

41 van Anders SM, Hampson E. Waist-to-hip ratio is positively associated with bioavailable testosterone, but negatively associated with sexual desire, in healthy adult premenopausal women. Psychosom Med 2005;67:241-5. 
Copyright of Journal of Sexual Medicine is the property of Blackwell Publishing Limited and its content may not be copied or emailed to multiple sites or posted to a listserv without the copyright holder's express written permission. However, users may print, download, or email articles for individual use. 